\title{
The Analysis of Formulaic Sequences in English Editorials from the Perspective of Theme Theory
}

\author{
Li huifeng, Liu Yuanyuan \\ School of Foreign Languages, Lanzhou University of Technology, Lanzhou 730050, China \\ scottlee78@126.com, sadieliu80@126.com
}

\begin{abstract}
The value of formulaic sequences can never be overestimated in second language acquisition. The present paper aims to analyze the distribution of different types of formulaic sequences in 30 randomly chosen English editorials from three modern English newspapers. The tool of the theme theory of Halliday is employed to reveal the distribution of these formulaic sequences in the theme/rheme of a sentence in English editorials. Among different categories, phrasal constraints dominate, followed by polywords and sentence builders. Formulaic sequences are of more value in theme than in rheme in English editorials in the writing and understanding of English editorials. theory

Index Terms - English editorials, formulaic sequences, theme
\end{abstract}

\section{Introduction}

Ever since the advent of Chomskyan competence, researches on second language acquisition have shifted attention to the importance of creative, rule-governed processes and to the systematic nature of language. The significance of formulaic sequences has been ignored. Formulaic sequence refers to a sequence, continuous or discontinuous, of words or other meaning elements stored or retrieved as a whole from memory at the time of use, rather than being subject to generation or analysis by language grammar. Many studies show formulaic sequences carry much importance in daily communication and in language for specific purpose, in which reduce the obstacles in language output. As one branch of ESP, news editorials can reveal the features of formulaic sequence to its fullest extent. This paper presents an overview of the relevant research of formulaic sequences and analyzes formulaic sequences in 30 English news editorials with Halliday's theme theory.

\section{Studies on Formulaic Sequences and Theme Theory}

\section{A. Studies on Formulaic Sequences}

The value of formulaic sequences in human communication and language acquisition could never be overestimated. The application of formulaic sequences, especially its application in second language acquisition, has been the focus of linguistic studies since 1980s. The latest and overall survey of formulaic language is provided by Alison Wray, who conducts a detailed survey to demonstrate that formulaic sequences are used in different ways by different kinds of speakers[1].

According to Nattinger and DeCarrico[2], formulaic sequences can be divided into four kinds: polywords are short phrases which function very much like individual lexical items; institutionalized expressions are lexical phrases of sentence length, usually functioning as separate utterances; phrasal constraints are short-to-medium-length phrases; sentence builders are lexical phrases that provide the framework for whole sentences.

\section{B. Studies on Theme Theory}

An equally important contribution to modern linguistics is the study of theme/rheme distribution by Prague School and Halliday. We suppose that in English, when we are trying to analyze how speakers or writers construct their message, the first, or more technically, the "left-most" constituent in each clause is of significance in that it "hooks" this clause onto the earlier ones and provides a starting point around which the remainder of the clause is constructed.[3]

Theme was later introduced by Halliday to be a notion in his theory, a "point of departure" as Halliday once called. [4] Halliday gave a comprehensive definition of the theme and rheme in his book An Introduction to Functional Grammar "the theme is the element which serves as the point of departure of the message; it is that with which the clause is concerned. The remainder of the message, the part in which the theme is developed, is called the rheme."[5]

\section{Corpus of the Present Study}

The data for investigation in the present paper consists of 30 news editorials collected from three influential English newspapers of English-speaking countries: The New York Times, Chicago Tribune and The Daily Telegraph.

In the first stage of data collection, a total number of 150 editorials are downloaded from the official websites of the three newspapers. The time span of the sample editorials is from January 1 to July 30, 2010. The materials to be investigated cover various fields. In the second stage of data collection, thirty editorials are randomly chosen from the downloaded 150 editorials, 10 from each of the three newspapers to constitute a corpus, with the total number of words amounting to 14,249 .

In the given corpus, the number of formulaic sequences, the number of different categories, and the percentage of those categories are illustrated in TABLE 1: 
TABLE1: Frequency of Formulaic Sequences in the Corpus

\begin{tabular}{|l|l|l|}
\hline Categories of FS & Frequency & Percentage \\
\hline Polywords & 88 & $24.4 \%$ \\
\hline Institutionalized expressions & 0 & $0 \%$ \\
\hline Phrasal constraints & 227 & $63.1 \%$ \\
\hline Sentence builders & 45 & $12.5 \%$ \\
\hline Total formulaic sequences & 360 & $100 \%$ \\
\hline
\end{tabular}

\section{The Distribution and Categories of Formulaic Sequences used in Theme and Rheme}

In order to reveal the features and functions of formulaic sequences in English editorials, the present paper conducts research on the distribution of formulaic sequences in the given corpus. Since the tool of thematic structure is employed, this paper puts more emphasis on the distribution of these formulaic sequences used in theme and rheme analyzed according to the theory of Halliday.

\section{A. The Distribution of Formulaic Sequences Used in Theme and Rheme}

In the corpus, a total number of 14,249 words of theme and rheme are recognized by the Wordcount of Microsoft word. According the Wordcount and the calculation of the author, the number of words in theme and rheme, the number of formulaic sequences in theme and rheme are listed in Table 2.

TABLE 2: Comparison of Formulaic Sequences and Word Number in Theme and Rheme

\begin{tabular}{|l|l|l|l|l|}
\hline & FS Number & PCT & Word number & PCT \\
\hline Theme & 100 & $27.8 \%$ & 2,915 & $20.5 \%$ \\
\hline Rheme & 260 & $72.2 \%$ & 11,334 & $79.5 \%$ \\
\hline Total number & 360 & $100 \%$ & 14,249 & $100 \%$ \\
\hline
\end{tabular}

As presented in TABLE 2, among the 14,249 English words in the corpus, only 2,915 , or $20.46 \%$, are used in theme, while the other 11,334 , or $79.54 \%$, words are used in rheme, i. e. an overwhelmingly majority of the words are employed in rheme. Due to the representativeness of the chosen language materials, a conclusion could be drawn that in modern English editorials, rheme or new information usually accounts for an overwhelming majority, about 80 percent, of the whole news reports. We can also easily see that although only $27.8 \%$ of the formulaic sequences are used in theme, this distribution should be considered with the fact that a much lower percentage, $20.5 \%$, of words are used in the part of theme. By contrast, even though the words used in rheme account for $79.5 \%$, the ratio of formulaic sequences used in rheme reaches a comparatively lower percentage, $72.2 \%$. The contrast could lead to the conclusion that the use of formulaic sequences is more obvious in theme that in rheme. factors:
Pinkerton (1988) maintains that by editorials, editors are mainly to state the news event, to offer background information and more importantly to extend moral judgment and to call for possible actions of the readers. The purposes of editorials just coincide with the functions of formulaic sequences. To reduce the time and effort of processing and to guarantee smooth communication, formulaic sequences could serve as social interaction devices, offer necessary topics and serve as discourse devices. More specifically, formulaic sequences could be used to introduce and shift a topic, to make an evaluation and a summon. These functions lead to the high frequency of formulaic sequences in English editorials.

On the other hand, according to Brown and Yule, in language discourse theme is mainly to connect back and link into the previous discourse, to maintain a coherent point of view and to serve as a point of departure for the further development of the discourse (1983:133). Theme is the common knowledge between speakers and listeners. Therefore, the more common knowledge speakers and listeners share, the fewer obstacles they will run into. Formulaic sequences are usually more easily acquired among all language learners and therefore they could to certain extent help reduce the obstacles in communication. This function of formulaic sequences is quite consistent with the function of theme in English. As a result, it is not surprising to find the comparatively high frequency of formulaic sequences in the part of theme.

\section{B. The Categories of Formulaic Sequences Used in Theme and Rheme}

According to Nattinger and DeCarrico, formulaic sequences can be further divided into four kinds. Accordingly, the 360 formulaic sequences sorted out in the corpus are divided into subcategories, and their respective percentages are calculated. Since in the given corpus, no institutionalized expressions are detected, all the formulaic sequences are divided into polywords, phrasal constraints and sentence builders. The number of formulaic sequences and the number of each categories used in theme and rheme are shown in TABLE 3.

TABLE 3: Categories of Formulaic Sequences in Theme and Rheme

\begin{tabular}{|l|l|l|l|l|}
\hline Types of FS & theme & percentage & rheme & percentage \\
\hline polywords & 34 & $34 \%$ & 54 & $21 \%$ \\
\hline phrasal constraints & 60 & $60 \%$ & 167 & $64 \%$ \\
\hline sentence builders & 6 & $6 \%$ & 39 & $15 \%$ \\
\hline total FS & 100 & $100 \%$ & 260 & $100 \%$ \\
\hline
\end{tabular}

1) The Distribution of Phrasal Constraints: As indicated in TABLE 3, the number of phrasal constraints in theme and rheme is greater than that of polywords and sentence builders, and the number in rheme is far greater than that in theme because phrasal constraints are more variable and flexible than polywords and sentence builders. They allow people to select various paradigmatic substitutions to fill in their slots, so this 
category of formulaic sequence can satisfy speakers and writers' needs to express different opinions. And rheme is the vector of new information. Editors mainly use rheme to give new information, so there are so many phrasal constraints used in rheme. Because of this, speakers or writers use more phrasal constraints in rheme to give information and express their own opinions. For example:

(1) While it can be hard to differentiate between criminal and military terrorism cases $(T) /$, this one was easy (R).

(2) And according to a study by the F.C.C. (T)/, the average download in the United States occurs at about half the advertised speed (R).

(3) The system, a sophisticated network of seismographs designed to collect data and feed it into analysis centers (T)/, has been running since $2006(\mathrm{R})$.

(4) But that (T)/ is not how it feels in Chicago, particularly in neighborhoods such as Chatham, which traditionally have been relatively safe $(R)$.

In the first two sentences, phrasal constraints "between...and..." and "according to" are used in theme. As maintained by Nattinger and DeCarrico, phrasal constraints are much more flexible and variable structures in the language command of most native speakers. Therefore, they are more frequently used in theme as the shared knowledge between speakers and listeners.

But in the last two sentences, the phrasal constraints "since..." and "such as..." are used in rheme of these sentences to indicate exemplification between different parts of the sentence. They are employed in the rheme part of the sentence to offer added information. Through the use of these formulaic sequences, for readers, the difficulty in the comprehension could be somehow effectively reduced.

2) The Distribution of Polywords: Polywords are short phrases which function much like individual lexical items that allow no variation and flexibility (Nattinger \& DeCarrio 2000: 37 ). Although they function as discourse devices, their quantity is small and their form is fixed. Because of this feature, it is easy to use them to perform a certain functions, such as expressing speaker or writer qualification of the topic, relating one topic to another, summarizing, shifting topics, and so on. For example:

(1) Meanwhile, businesses(T)/ continue to lay off workers, which means that more newly poor people will seek coverage from Medicaid, putting an even greater strain on state budgets when they are least able to afford it (R).

(2) Of course, there(T)/ is also an upside to having 71 million flu shots sitting on the shelf (R).

(3) Obama(T)/, by contrast, has embraced policies more ambitious and sometimes quite different from what he led Americans to expect — and which may not work out so well (R).

(4) There (T)/ won't be any such triumphant announcements from President Barack Obama, at least not soon (R).
In the first two examples, polywords are used in theme. "Meanwhile" is used as a device for topic shift under the condition that both the topic discussed and the one to be discussed are known to both the speaker and the listener. "Of course" is used to illustrate the assertive attitude of the speaker. Since it is used in the theme part of the sentence, there must be clue for readers to know the writer's attitude prior to this formulaic sequence.

A quite obvious example could be offered in the fourth sentence in that "sometimes" occurs in the rheme of the sentence as new information to tell readers that only some of promises have not been kept. "At least" is used in the rheme parts of the sentences to serve as devices for new information complement. It can reveal the attitude of speakers and help present emphasized information.

\section{The Distribution of Sentence Builders}

Compared with the two types of formulaic sequences above, sentence builders function as the frames and macroorganizers of discourses. And they are indicators of logical and orderly writing. But in most cases, the theme of one sentence is shorter than rheme. That means the theme of one sentence may not be a complete sentence, unless the sentence is a clause complex. So the number of sentence builders used in theme is far less than that used in rheme.

(1) If there was any doubt about the wisdom of this approach (T)/, it was dispelled by a letter to The Daily Telegraph signed by 23 leading businessmen (R).

(2) After rosy promises last spring that there'd be enough vaccine to go around (T)/, the feds backtracked again and again $(\mathrm{R})$.

(3) Since this happened some distance from hussain's home (T)/, it is likely that he would have failed the "grossly disproportionate" test (R).

(4) While this may be a technical matter $(\mathrm{T}) /$, it is indicative of a carelessness that cannot be brushed aside on the grounds that stopping suspected terrorists is crucial to our general security $(\mathrm{R})$.

In the first two sentences, sentence builders are used in theme in the case that these formulaic sequences are used in the subordinate clause. According to Halliday, under this condition, the whole sentence can be classified as the part of theme. In the third and last example, "it is likely that" and "on the grounds that" are use in rheme to offer new information to the anticipation of the speaker and the condition for the main clause "it is indicative of a carelessness that cannot be brushed aside".

To sum up, among the three categories of formulaic sequences, the frequency of phrasal constraints in editorials is higher than other two categories of formulaic sequences, followed by polywords, sentence builders. With a better understanding of formulaic sequences, we can not only have a better comprehension but also improve the quality of English editorials. 


\section{Conclusion}

The use of formulaic sequences could not only enhance daily communication but also reduce the obstacle in the understanding of English editorials. From the analysis of the present thesis, English editorials writers and readers could get to know firstly what type of formulaic sequences to use and secondly in which part of a sentence to use these formulaic sequences in English editorials.

Among the four categories of formulaic sequences, the number of phrasal constraints predominates in the corpus. The comparatively higher frequency of this category results from its higher level of flexibility and variation. Second to the use of phrasal constraints is the use of polywords because one basic function of polywords is discourse device. The employment of polywords could effectively guarantee the coherence of English editorials. Sentence builders come at the end of the list mainly because the theme of one sentence may not be a complete sentence, unless the sentence is a clause complex, thus the number of them is the smallest. Institutionalized expressions are not found in the corpus. And the ratio of repeated phrasal constraints is higher than that of repeated polywords and sentence builders.

As for the distribution of formulaic sequences in theme and rheme in the corpus, though the number of formulaic sequences in theme of the corpus account for only $27.8 \%$, the figure should be considered with the fact that the word number of theme account for only $20.5 \%$ of the total language materials. Given the lower ratio of the total words in theme, the comparative low ratio of the formulaic sequences used in theme can still speak of the significant function of formulaic sequences to guarantee the smoothness of communication in English editorials. Besides, the use of different formulaic sequences in theme is more significant than that in rheme.

\section{Acknowledgment}

Special gratitude should be addressed to Professor Shi Shunliang from School of Foreign languages, Lanzhou University of Technology, who offered much guidance and help for this paper.

\section{Reference}

[1] A. Wray, Formulaic Language and the Lexicon. Cambridge: Cambridge University Press, 2002.

[2] J. R .Nattinger, and J. S. DeCarrico. Lexical Phrases and Language Teaching. Shanghai: Shanghai Foreign Language Education Press, 2000.

[3] G. Brown and G. Yule, Discourse Analysis Beijing: Foreign Language Teaching and Research Press, 2000.

[4] M.A.K. Halliday, An Introduction to Functional Grammar. Beijing: Foreign Language Teaching and Research Press, 2000.

[5] M.A.K. Halliday, Explorations in the Functions of Language. London: Edward Arnold, 1977. 